\title{
Current Response in Extended Systems as a Geometric Phase: Application to Variational Wavefunctions
}

\author{
Balázs HeTÉNYI ${ }^{1,2}$ \\ ${ }^{1}$ Department of Physics, Bilkent University, 06800 Ankara, Turkey \\ ${ }^{2}$ Institute for Theoretical Physics/Computational Physics, Graz University of Technology, \\ Petersgasse 16, Graz, A-8010, Austria
}

(Received July 21, 2012; accepted October 3, 2012; published online November 28, 2012)

\begin{abstract}
The linear response theory for current is investigated in a variational context. Expressions are derived for the Drude and superfluid weights for general variational wavefunctions. The expression for the Drude weight highlights the difficulty in its calculation since it depends on the exact energy eigenvalues which are usually not available in practice. While the Drude weight is not available in a simple form, the linear current response is shown to be expressible in terms of a geometric phase, or alternatively in terms of the expectation value of the total position shift operator. The contribution of the geometric phase to the current response is then analyzed for some commonly used projected variational wavefunctions (Baeriswyl, Gutzwiller, and combined). It is demonstrated that this contribution is independent of the projectors themselves and is determined by the wavefunctions onto which the projectors are applied.
\end{abstract}

KEYWORDS: DC conductivity, superfluid weight, geometric phase

\section{Introduction}

Variational studies have contributed greatly to our understanding of correlated systems. In part this is due to their relative simplicity, applicability to larger sizes irrespective of the number of dimensions, and the easily accessible physical insight they provide. In the case of the Hubbard model ${ }^{1-4)}$ frequently used variational wavefunctions include the Gutzwiller ${ }^{1,2)}$ (GWF) and Baeriswyl wavefunctions ${ }^{5-7)}$ (BWF), and their combinations. The former is based on suppressing charge fluctuations in the noninteracting solution, the latter on projecting with the hopping operator onto a wavefunction in the large interaction limit.

The GWF has been studied by a variety of methods. It can be solved exactly in one ${ }^{8,9)}$ and infinite ${ }^{9-11)}$ dimensions, and it can be simulated in two and three dimensions by variational Monte Carlo. ${ }^{12}$ The one-dimensional exact solution produces a state with a finite discontinuity of the momentum density at the Fermi surface. Millis and Coppersmith $^{13)}$ have investigated the response of the GWF and have concluded that it is metallic with a conductivity proportional to the kinetic energy. Insulating behavior in projected wavefunctions similar to the one due to Gutzwiller can be produced by generalized projection operators, ${ }^{14,15)}$ for example non-centro-symmetric or singular projectors.

Calculating the Drude or the superfluid weight in a variational context is a difficult issue. These two quantities can be cast in terms of identical expressions [see eq. (1)], the second derivative of the ground state energy with respect to a Peierls phase. ${ }^{16-19)}$ As pointed out by Scalapino, White, and Zhang, the two quantities differ in the interpretation of the derivative. ${ }^{17,18)}$ For the Drude weight the Peierls phase shifts the ground state energy adiabatically, remaining always in the same state, for the superfluid weight level crossings are also considered.

In this work general expressions for the Drude and superfluid weights are derived in a variational setting. For the Drude weight deriving an easily applicable expression is a difficult issue, since the expression derived herein depends on the exact eigenvalues of the perturbed Hamiltonian, in practical settings often not available. It is then demonstrated that the linear response expression for the current can be cast in terms of a geometric phase. The tool for calculating this geometric phase (the total position shift operator) are also presented. The formalism is then used to interpret the current response of projected wavefunctions. It is demonstrated that the current response in the commonly used Gutzwiller and Baeriswyl projected, as well as wavefunctions based on combinations of the two projections, produce a current response identical to the wavefunction on which the projections are applied (the Fermi sea or the wavefunction in the strongly interacting limit).

\section{Drude and Superfluid Weights in Variational Theory}

An expression for the frequency $(\omega)$ and wave vector (q)-dependent conductivity was derived by Kohn. ${ }^{16)}$ The DC conductivity (Drude weight, $D_{c}$ ) corresponds to the strength of the $\delta$-function peak of the conductivity in the zero frequency limit. The correct expression for $D_{c}$ is obtained by first taking the limit $(\mathbf{q} \rightarrow 0)$ and then the other limit $\omega \rightarrow 0$. $D_{c}$ is often expressed ${ }^{16,19)}$ in terms of the second derivative of the ground state energy with respect to a phase associated with the perturbing field as

$$
D_{c}=\frac{\pi}{L}\left[\frac{\partial^{2} E_{0}(\Phi)}{\partial \Phi^{2}}\right]_{\Phi=0} .
$$

Here $E_{0}(\Phi)$ denotes the perturbed ground state energy, $\Phi$ denotes the Peierls phase.

Scalapino, White, and Zhang (SWZ) ${ }^{17,18)}$ have investigated the distinction between the Drude and superfluid weights. In particular they studied the importance of the order of different limits $(\omega \rightarrow 0, \mathbf{q} \rightarrow 0)$ for the conductivity. In a variational context implementation of the frequency limit is not straightforward, since, strictly speaking there is no frequency to speak of. However, SWZ have also pointed out that the derivative with respect to the phase $\Phi$ in eq. (1) is ambiguous. They showed that if the derivative is defined via adiabatically shifting the state which is the ground state at zero field, then the Drude weight results. In the presence 
of level crossings the adiabatically shifted state may be an excited state for a finite value of the perturbation. The superfluid weight is obtained if the derivative corresponds to the "envelope function", i.e., the ground state of the perturbed system is taken to define the derivative. The distinction between these two derivatives can be implemented by embedding the periodic system under study in a larger periodic system, and defining the perturbation in terms of the periodic boundary conditions of this larger system. In cases in which level crossings are close to $\Phi=0$ conductors, superconductors, and insulators can be distinguished. ${ }^{17,18,20)}$ In general, the position of level crossings depends on dimensionality. ${ }^{17,18)}$

The finite temperature extension of $D_{c}$ has been given by Zotos, Castella, and Prelovšek ${ }^{21)}$ (ZCP). This generalization can be summarized as

$$
D_{c}(T)=\frac{\pi}{L} \sum_{n} \frac{\exp \left(-\beta E_{n}\right)}{Q}\left[\frac{\partial^{2} E_{n}(\Phi)}{\partial \Phi^{2}}\right]_{\Phi=0} .
$$

Note in this expression the Boltzmann weight factors remain unchanged as the perturbation $\Phi$ is turned on. Equation (2) has been applied ${ }^{22)}$ to calculate the DC conductivity in strongly correlated systems. Taking the zero temperature limit reproduces Kohn's expression for $D_{c}$. To define a finite temperature analog of $D_{s}$ one lets the Boltzmann weight factors depend on the perturbing field $\Phi$ as

$$
D_{s}(T)=\frac{\pi}{L}\left[\frac{\partial^{2}}{\partial \Phi^{2}} \sum_{n} \frac{\exp \left(-\beta E_{n}(\Phi)\right)}{Q} E_{n}(\Phi)\right]_{\Phi=0} .
$$

Indeed the ground state superfluid weight is reproduced in the zero temperature limit. Equations (2) and (3) follow from the assumption that the distinction between the Drude and superfluid weights is due to the different types of derivatives as discussed by SWZ.

Similarly, in deriving expressions for $D_{c}$ and $D_{s}$ in a variational setting our starting assumption will also be that the distinction between the two quantities is due to the effects of level crossings. Suppose $|\tilde{\Psi}(\gamma)\rangle$ is a variational wavefunction, where $\gamma$ denotes a set of variational parameters, which we wish to use to optimize some Hamiltonian $\hat{H}$ with eigenbasis

$$
\hat{H}\left|\Psi_{n}\right\rangle=E_{n}\left|\Psi_{n}\right\rangle .
$$

The estimate for the ground state energy may be written in terms of a density matrix as

$$
\begin{aligned}
\langle\tilde{\Psi}(\gamma)|\hat{H}| \tilde{\Psi}(\gamma)\rangle & =\sum_{n}\left\langle\tilde{\Psi}(\gamma) \mid \Psi_{n}\right\rangle E_{n}\left\langle\Psi_{n} \mid \tilde{\Psi}(\gamma)\right\rangle \\
& =\sum_{n} P_{n} E_{n}
\end{aligned}
$$

the probabilities can be written as

$$
P_{n}=\left|\left\langle\tilde{\Psi}(\gamma) \mid \Psi_{n}\right\rangle\right|^{2} \text {. }
$$

Comparing with eq. (2) it is obvious that a consistent formalism requires that the variational Drude weight be defined as

$$
D_{c}=\frac{\pi}{L} \sum_{n} P_{n} \frac{\partial^{2} E_{n}(\Phi)}{\partial \Phi^{2}},
$$

with $P_{n}$ independent of the perturbation $\Phi$. It follows that the variational parameter $\gamma$ is independent of the perturbation $\Phi$.
The variational analog of $D_{s}$ [based on eq. (3)] corresponds to

$$
D_{s}=\frac{\pi}{L} \frac{\partial^{2}}{\partial \Phi^{2}} \sum_{n} P_{n}(\Phi) E_{n}(\Phi),
$$

where the probabilities $P_{n}(\Phi)$ depend on $\Phi$ and the variational parameters $\gamma$ in this case also depend on $\Phi$.

A central difficulty in calculating $D_{c}$ in a variational theory is the fact that it depends on the exact eigenvalues of the perturbed Hamiltonian [see eq. (1)], however variational theories are usually applied in cases where the exact solution is not easily accessible. While the Drude weight remains a difficult problem in general, it is shown below that the current can be cast in terms of a geometric phase, and evaluated even in a variational context.

\section{Current in Terms of a Geometric Phase}

In this section we consider the adiabatic current response of a system in general, not only in a variational context. After showing that the persistent current can be expressed as a geometric phase, ${ }^{23,24)}$ we explicitly construct the mathematical tools to calculate it, and use the results in the next section to interpret the GWF. Since the current can be cast in terms of observables, it follows that the calculation of the Drude weight is also accessible, being the first derivative of the current as a function of the Peierls phase.

Consider a system periodic in $L$, and experiencing a perturbation in the form of a Peierls phase $\Phi$. Its Hamiltonian can be written as

$$
\hat{H}(\Phi)=\sum_{i=1}^{N} \frac{\left(\hat{p}_{i}+\Phi\right)^{2}}{2 m}+V\left(x_{1}, \ldots, x_{N}\right) .
$$

The following identity also holds

$$
\partial_{\Phi} \hat{H}(\Phi)=\sum_{i=1}^{N} \frac{\left(\hat{p}_{i}+\Phi\right)}{m} .
$$

The ground state energy can be written as

$$
E(\Phi)=\langle\Psi(\Phi)|\hat{H}(\Phi)| \Psi(\Phi)\rangle .
$$

The average current for such a system can be expressed as ${ }^{16)}$

$$
J(\Phi)=\partial_{\Phi} E(\Phi)=\left\langle\Psi(\Phi)\left|\partial_{\Phi} \hat{H}(\Phi)\right| \Psi(\Phi)\right\rangle .
$$

Substituting for the partial derivative of the Hamiltonian we obtain

$$
J(\Phi)=\frac{N \Phi}{m}+\sum_{i=1}^{N}\left\langle\Psi(\Phi)\left|\frac{\hat{p}_{i}}{m}\right| \Psi(\Phi)\right\rangle .
$$

In the position representation the current can be written

$$
J(\Phi)=\frac{N \Phi}{m}-\frac{\mathrm{i}}{m} \sum_{i=1}^{N}\left\langle\Psi(\Phi)\left|\frac{\partial}{\partial x_{i}}\right| \Psi(\Phi)\right\rangle .
$$

Next we rewrite the wavefunction in terms of a shift of the total position and define a wavefunction

$$
\left\langle x_{1}, \ldots, x_{N} \mid \Psi(\Phi ; X)\right\rangle=\Psi\left(x_{1}+X, \ldots, x_{N}+X ; \Phi\right) .
$$

The action of the total momentum can then be cast in terms of the derivative with respect to $X$ as

$$
\begin{aligned}
& \sum_{i=1}^{N} \frac{\partial}{\partial x_{i}} \Psi\left(x_{1}+X, \ldots, x_{N}+X ; \Phi\right) \\
& \quad=\partial_{X} \Psi\left(x_{1}+X, \ldots, x_{N}+X ; \Phi\right) .
\end{aligned}
$$


The effect of $X$ on the particle positions is similar to the effect of the Peierls phase on the momenta. Like the Peierls phase it is an external parameter, so one can perform adiabatic cycles as a function of it. Averaging in $X$ over the unit cell $\frac{1}{L} \int_{0}^{L} \mathrm{~d} X \ldots$ leads to

$$
J(\Phi)=\frac{N \Phi}{m}-\frac{\mathrm{i}}{m L} \int_{0}^{L} \mathrm{~d} X\left\langle\Psi(\Phi ; X)\left|\partial_{X}\right| \Psi(\Phi ; X)\right\rangle .
$$

The second term in eq. (17) is a geometric phase. ${ }^{23)}$ Since it results from the periodicity of the parameter $X$ it is similar to the geometric phase derived by Zak. ${ }^{25)}$ It is also similar to the geometric phase expression appearing in the modern theory of polarization, ${ }^{26)}$ with the variable $X$ playing the role of the crystal momentum in this case. Thus the current due to a perturbation can be expressed in terms of a constant proportional to the number of particles, and a geometric phase term. Below an interpretation of the phase term is given. It is interesting to note that a finite persistent current is in principle possible for an unperturbed system (the case $\Phi=0)$.

The next question to address is the actual calculation of this quantity. We can construct a scheme which is in the spirit of the total position operator proposed by Resta ${ }^{27,28)}$ to calculate the polarization. We consider the case $\Phi=0$ (and suppress the notation), without loss of generality. We first rewrite the Berry phase appearing in the expression for the current in terms of its discretized analog as ${ }^{29)}$

$$
J(0)=\lim _{\Delta X \rightarrow 0} \frac{1}{m L} \operatorname{Im} \ln \prod_{s=0}^{M-1}\langle\Psi(s \Delta X) \mid \Psi((s+1) \Delta X)\rangle .
$$

The continuous expression can be recovered by Taylor expanding the wavefunction $|\Psi((s+1) \Delta X)\rangle$ around $s \Delta X$ and taking the limit as $\Delta X \rightarrow 0$. Indeed

$$
\begin{aligned}
J(0)= & \lim _{\Delta X \rightarrow 0} \frac{1}{m L} \operatorname{Im} \sum_{s=0}^{M-1} \ln \langle\Psi(s \Delta X) \mid[\mid \Psi(s) \Delta X)\rangle \\
& \left.+\partial_{X}|\Psi(s \Delta X)\rangle \Delta X\right] \\
= & \ln \left[1+\left\langle\Psi(s \Delta X)\left|\partial_{X}\right| \Psi(s \Delta X)\right\rangle \Delta X\right] .
\end{aligned}
$$

When the limit $\Delta X \rightarrow 0$ is taken the natural logarithm can be expanded and we obtain

$$
\begin{aligned}
J(0) & =\frac{1}{m L} \operatorname{Im}\left[\int \mathrm{d} X\left\langle\Psi(X)\left|\partial_{X}\right| \Psi(X)\right\rangle\right] \\
& =-\frac{\mathrm{i}}{m L} \int \mathrm{d} X\left\langle\Psi(X)\left|\partial_{X}\right| \Psi(X)\right\rangle .
\end{aligned}
$$

The shift in the total position of the system by a value of $\Delta X$ can be accomplished using the total position shift operator $\hat{U}(\Delta X)$. The explicit form of this operator will be derived below, for now we assume its existence. We define it as

$$
\hat{U}(\Delta X)|\Psi(X)\rangle=|\Psi(X+\Delta X)\rangle .
$$

Using eq. (21) we can express the product in eq. (18) as

$$
\prod_{s=0}^{M-1}\langle\Psi(s \Delta X) \mid \Psi((s+1) \Delta X)\rangle=\langle\Psi(0)|\hat{U}(\Delta X)| \Psi(0)\rangle^{M} \text {. }
$$

Substituting into eq. (18) the expression for the current becomes

$$
J(0)=\lim _{\Delta X \rightarrow 0} \frac{1}{m} \frac{1}{\Delta X} \operatorname{Im} \ln \langle\Psi(0)|\hat{U}(\Delta X)| \Psi(0)\rangle .
$$

The total position shift operator can be constructed using real space permutation operators. This derivation has been given elsewhere, ${ }^{30)}$ here we emphasize the main results. In second quantized notation the permutation operator between two positions can be written as

$$
P_{i j}=1-\left(c_{i}^{\dagger}-c_{j}^{\dagger}\right)\left(c_{i}-c_{j}\right)
$$

This operator has the properties

$$
P_{i j} c_{j}=c_{i} P_{i j}, P_{i j} c_{i}=c_{j} P_{i j}, P_{i j} c_{j}^{\dagger}=c_{i}^{\dagger} P_{i j}, P_{i j} c_{i}^{\dagger}=c_{j}^{\dagger} P_{i j} \text {. }
$$

Assuming a grid with spacing $\Delta X$, using $P_{i j}$ we can construct an operator which shifts all the positions on the grid in a periodic system. The operator

$$
\hat{U}(\Delta X)=P_{12} P_{23} \cdots P_{L-1 L},
$$

where it is assumed that the indices refer to particular grid points, has the property that

$$
\hat{U}(\Delta X) c_{i}= \begin{cases}c_{i-1} \hat{U}(\Delta X), & i=2, \ldots, L \\ c_{L} \hat{U}(\Delta X), & i=1 .\end{cases}
$$

It also holds that

$$
\hat{U}(\Delta X) \tilde{c}_{k}=\mathrm{e}^{\mathrm{i} \Delta X k} \tilde{c}_{k} \hat{U}(\Delta X),
$$

where $\tilde{c}_{k}$ denotes the annihilation operator in reciprocal space. Equation (28) can be demonstrated by Fourier transforming $\tilde{c}_{k}$ and applying (27). Taking the Fermi sea

$$
|\mathrm{FS}\rangle=\tilde{c}_{k_{1}}^{\dagger} \cdots \tilde{c}_{k_{N}}^{\dagger}|0\rangle,
$$

as an example one can show that

$$
\hat{U}(\Delta X)|\mathrm{FS}\rangle=\mathrm{e}^{\mathrm{i} \Delta X K}|\mathrm{FS}\rangle,
$$

with $K=\sum_{i=1}^{N} k_{i}$.

As an example we consider again the non-interacting Fermi sea given by

$$
|\mathrm{FS}\rangle=\tilde{c}_{k_{1}}^{\dagger} \cdots \tilde{c}_{k_{N}}^{\dagger}|0\rangle,
$$

where the $k$-vectors are spread symmetrically around zero. Applying a perturbation $\Phi$ shifts all $k$-vectors by $\Phi$. The resulting current is

$$
J(\Phi)=\frac{2 N}{m} \Phi
$$

corresponding to a Drude weight of $D_{c}=2 N / m$. It is interesting to see that the current is proportional to twice the number of particles. In a Fermi sea conduction can occur due to particles as well as holes, of which at halffilling there are an equal number. For systems with bound particles and holes, $J(\Phi)$ is reduced, as bound excitons do not participate in conduction and reduce the effective number of charge carriers. Thus the geometric phase in eq. (17) accounts for exciton binding. When all the particles are bound to holes then the constant term in eq. (17) is cancelled by the phase term leading to $J(\Phi)=0$. An example of bound particles and holes in the same band leading to insulating behaviour is the Baeriswyl variational wavefunction. ${ }^{6)}$

\section{Contribution of the Geometric Phase to the Current Response of Projected Wavefunctions}

In this section we provide the response theory of some commonly used projected wavefunctions. ${ }^{1,2,5,6)} \mathrm{We}$ empha- 
size that it is the contribution of the phase term to the current response we calculate, not the Drude weight, which is the first derivative of the current response with respect to the perturbing phase.

The Gutzwiller wavefunction ${ }^{1,2)}$ (GWF) was proposed as a variational wavefunction for the Hubbard model, and it has the form

$$
\left|\Psi_{\mathrm{G}}(\gamma)\right\rangle=\mathrm{e}^{-\gamma \hat{D}}|\mathrm{FS}\rangle,
$$

where $\hat{D}=\sum_{i} n_{i \uparrow} n_{i \downarrow}$. Without loss of generality we consider the one-dimensional case.

Before developing the current response theory of the GWF, we present the calculation of a quantity which expresses the extent of localization. Localization has been suggested long ago as a general criterion of metallicity, ${ }^{16)}$ and the relation of the spread to the DC conductivity has been shown in a number of places. ${ }^{20,27,28)}$ In particular we calculate the normalized spread defined as

$$
\frac{\left\langle X^{2}\right\rangle-\langle X\rangle^{2}}{L^{2}} .
$$

Due to the ill-defined nature of the position operator in periodic systems we choose a sawtooth representation which can be written as

$$
X=\sum_{\substack{m=-L / 2 \\ m \neq 0}}^{L / 2-1}\left(\frac{1}{2}+\frac{\hat{W}^{m}}{\exp \left(\mathrm{i} \frac{2 \pi m}{L}\right)-1}\right),
$$

where $\hat{W}$ denotes the total momentum shift operator, which has the property that

$$
\hat{W}|\Psi(K)\rangle=|\Psi(K+(2 \pi) / L)\rangle .
$$

The construction ${ }^{31)}$ of this operator is analogous to the total position shift operator used to define the persistent current in $\S 3$. For a state $|\tilde{\Phi}\rangle$ diagonal in the position representation one can write

$$
\hat{W}|\tilde{\Phi}\rangle=\exp \left(\mathrm{i} \frac{2 \pi}{L} \sum_{i} \hat{x}_{i}\right)|\tilde{\Phi}\rangle,
$$

where $x_{i}$ denotes the position of particle $i$. Using the sawtooth representation one can show that for the Fermi sea that the spread in position

$$
\frac{\left\langle X^{2}\right\rangle-\langle X\rangle^{2}}{L^{2}}=\lim _{L \rightarrow \infty} \frac{1}{L^{2}} \sum_{m=1}^{L-1} \frac{1}{2\left(1-\cos \left(\frac{2 \pi m}{L}\right)\right)}=\frac{1}{12} \text {. }
$$

To show this one needs to substitute eq. (35) into eq. (34), and then use the fact that

$$
\hat{W}^{m}|\mathrm{FS}\rangle=\left\{\begin{array}{ll}
0 & m=2, \ldots, L-1 \\
|\mathrm{FS}\rangle & m=0
\end{array} .\right.
$$

Our results are shown in Table I. The GWF results for two different values of the variational parameter were calculated for a one-dimensional system based on the variational Monte Carlo method of Yokoyama and Shiba. ${ }^{12)}$ The fact that the normalized spread approaches a constant for large $L$ (system size) indicates that the system is delocalized, hence metallic. What is surprising in these results, however, is that for large $L$ the spread of all three examples converges to the same value. The projecting out of double occupations in the GWF
Table I. Spread in the total position divided by the square of the system size for the Fermi sea and the Gutzwiller wavefunction. Two different values of the variational parameter, $\gamma=1.0$ and 2.0 are shown. As the system size increases the value $1 / 12$ is approached by all three systems. The approach to the limiting value slows down as correlation effects are introduced, it is slowest for $\gamma=2.0$, the "most projected" of the three examples.

\begin{tabular}{cccc}
\hline$L$ & Fermi sea & $\gamma=1.0$ & $\gamma=2.0$ \\
\hline 12 & 0.08275 & $0.079(1)$ & $0.0412(9)$ \\
24 & 0.08312 & $0.0830(6)$ & $0.0682(6)$ \\
36 & 0.08327 & $0.0831(5)$ & $0.0797(5)$ \\
48 & 0.08330 & $0.0833(4)$ & $0.0824(4)$ \\
60 & 0.08331 & $0.0829(3)$ & $0.0830(3)$ \\
$\infty$ & $1 / 12$ & & \\
\hline
\end{tabular}

seems to have no effect on the spread for large $L$, and is identical to the result for the Fermi sea. The GWF though is thought to be a representative of "bad metals", metals whose conductivity is reduced due to strong correlations. ${ }^{32,33)}$

It turns out that these results are actually consistent with what one obtains for the current response. We consider the phase term under a perturbation in the form in eq. (23). Consider first the action of the operator $\hat{U}(\Delta X)$ on the GWF.

$$
\hat{U}(\Delta X)|\Psi(\gamma)\rangle=\hat{U}(\Delta X) \mathrm{e}^{-\gamma \sum_{i} n_{i \uparrow} n_{i \downarrow}}|\mathrm{FS}\rangle .
$$

The operator $\hat{U}(\Delta X)$ shifts the positions of all particles by one lattice spacing. Such a shift will not change the total number of double occupations, hence the Gutzwiller projector and the total position shift operator commute. We can write

$$
\begin{aligned}
\hat{U}(\Delta X)|\Psi(\gamma)\rangle & =\mathrm{e}^{-\gamma \sum_{i} n_{i \uparrow} n_{i \downarrow}} \hat{U}(\Delta X)|\mathrm{FS}\rangle \\
& =\mathrm{e}^{\mathrm{i} \Delta X \sum_{i} k_{i}}|\Psi(\gamma)\rangle,
\end{aligned}
$$

where $\sum_{i} k_{i}$ denote the sum over the momenta of the Fermi sea. One obtains exactly the same result in the absence of the Gutzwiller projector. When substituting back into eq. (23) we find that the current response of the GWF is exactly that of the Fermi sea, and this result is independent of correlations (whose strength increases monotonically with the variable $\gamma$ ). The above derivation can be extended to projections based on Jastrow-type correlations and the conclusion is valid as long as the projections are centrosymmetric (considered in ref. 13). It has been shown ${ }^{14)}$ that non-centrosymmetric correlators can produce an insulating state. The current response in this case will also not follow the derivation above, since a shift in all the particles can change the contribution to the projector. Another exception is the case when $\gamma \rightarrow \infty$, i.e., the singular case, which in general is also known to allow for insulating behavior. ${ }^{15)}$

For the GWF one can obtain further insight into the current response by writing it in the position representation as

$$
\left|\Psi_{\mathrm{G}}\right\rangle=\sum_{\mathbf{R}} \mathrm{e}^{-\gamma D(\mathbf{R})} \operatorname{Det}(\mathbf{K} ; \mathbf{R})|\mathbf{R}\rangle .
$$

In eq. (42) $\mathbf{R}$ indicates the configurations of particles (both up-spin and down-spin), $D(\mathbf{R})$ indicates the number of double occupations for a particular configuration of particles, $\operatorname{Det}(\mathbf{K} ; \mathbf{R})$ denotes the product of Slater determinants for up-spin and down-spin electrons, and $|\mathbf{R}\rangle$ denotes a position space eigenstate. From eq. (42), we see that the projection changes the relative weight of different config- 
urations but leaves their phases intact. ${ }^{33)}$ The fact that the current, a quantity related to the phase of the wavefunction, is unaltered by the Gutzwiller projection coincides with the result above, namely that the persistent current for a Gutzwiller wavefunction is determined exclusively by the Fermi sea. In fact Millis and Coppersmith ${ }^{13)}$ suggest a scheme in which a projector operator of the form $\mathrm{e}^{\mathrm{i} S}$, with $S=(1 / U)\left(H_{t}^{+}-H_{t}^{-}\right),\left[H_{t}^{+}\left(H_{t}^{-}\right)\right.$raises (lowers) the number of double occupations] acts on the Fermi sea to produce an insulating wavefunction. Clearly this scheme would alter the phases of the Fermi sea.

The above reasoning can be extended to other commonly used projected variational wavefunctions. The BaeriswylGutzwiller wavefunction can be written

$$
\left|\Psi_{\mathrm{BG}}(\alpha, \gamma)\right\rangle=\mathrm{e}^{-\alpha \hat{T}} \mathrm{e}^{-\gamma \hat{D}}|\mathrm{FS}\rangle .
$$

In this case $\hat{T}$ denotes the kinetic energy, and $\alpha$ denotes the variational parameter. Since the total position shift operator $\hat{U}(\Delta X)$ is diagonal in momentum space, it trivially commutes with the projector $\mathrm{e}^{-\alpha \hat{T}}$. We conclude that the current response of the Baeriswyl-Gutzwiller projected wavefunction is identical to that of the Fermi sea. The other two commonly used variational wavefunctions are the Baeriswyl and Gutzwiller-Baeriswyl projected wavefunctions. Their form is

$$
\begin{aligned}
\left|\Psi_{\mathrm{B}}(\alpha, \gamma)\right\rangle & =\mathrm{e}^{-\alpha \hat{T}}\left|\Psi_{\infty}\right\rangle, \\
\left|\Psi_{\mathrm{GB}}(\alpha, \gamma)\right\rangle & =\mathrm{e}^{-\gamma \hat{D}} \mathrm{e}^{-\alpha \hat{T}}\left|\Psi_{\infty}\right\rangle .
\end{aligned}
$$

In eqs. (44) and (45) $\left|\Psi_{\infty}\right\rangle$ denotes the wavefunction in the limit of infinite interaction. This function is in general not known. Again one can exploit the fact that the total position shift commutes with the projector operators and conclude that the current response in both cases will depend on $\left|\Psi_{\infty}\right\rangle$ exclusively. While this function is not known, in general, in the half-filled case one can assume that its current response is zero.

\section{Conclusion}

The current response was investigated in the context of variational theory. The Drude and superfluid weights have seemingly identical expressions (second derivative of the ground state energy with respect to the Peierls phase), however, as was pointed by Scalapino, White, and Zhang, the meaning of the derivative differs between the two, one being the adiabiatic the other the "envelope" derivative. Assuming their interpretation of the derivative we derived the expressions for the Drude and superfluid weights appropriate for variational theory. A key difficulty with the former is the appearance of the exact eigenstates of the perturbed Hamiltonian, in general not available in practical situations where variational theory is used. As a partial remedy the persistent current was shown to consist of a constant term, proportional to the perturbation and the number of charge carriers, and a geometric phase term. This expression can be used in practical settings to obtain the Drude weight by numerically taking the first derivative of the current with respect to the phase. The current response of several commonly used variational wavefunctions was also analyzed, and shown that variational wavefunctions which use a Baeriswyl or Gutzwiller projection will have a current response determined by the wavefunction on which the projectors are applied (Fermi sea or the solution in the strongly interacting limit).

\section{Acknowledgements}

This work was supported by the Turkish agency for basic research (TÜBITAK, grant No. 112T176). Part of the work was carried out at the Graz University of Technology under a grant from FWF (No. P21240-N16). The author is grateful to the Physical Society of Japan for financial support in publication.

1) M. C. Gutzwiller: Phys. Rev. Lett. 10 (1963) 159.

2) M. C. Gutzwiller: Phys. Rev. 137 (1965) A1726.

3) J. Hubbard: Proc. R. Soc. A 276 (1963) 238.

4) J. Kanamori: Prog. Theor. Phys. 30 (1963) 275.

5) D. Baeriswyl: in Nonlinearity in Condensed Matter, ed. A. R. Bishop, D. K. Campbell, D. Kumar, and S. E. Trullinger (Springer, Heidelberg, 1986).

6) D. Baeriswyl: Found. Phys. 30 (2000) 2033.

7) M. Dzierzawa, D. Baeriswyl, and L. M. Martelo: Helv. Phys. Acta 70 (1997) 124.

8) W. Metzner and D. Vollhardt: Phys. Rev. Lett. 59 (1987) 121.

9) W. Metzner and D. Vollhardt: Phys. Rev. Lett. 62 (1989) 324.

10) W. Metzner and D. Vollhardt: Phys. Rev. B 37 (1988) 7382.

11) W. Metzner and D. Vollhardt: Helv. Phys. Acta 63 (1990) 364.

12) H. Yokoyama and H. Shiba: J. Phys. Soc. Jpn. 56 (1987) 1490.

13) A. J. Millis and S. N. Coppersmith: Phys. Rev. B 43 (1991) 13770.

14) M. Capello, F. Becca, M. Fabrizio, S. Sorella, and E. Tosatti: Phys. Rev. Lett. 94 (2005) 026406.

15) M. Capello, F. Becca, M. Fabrizio, S. Sorella, and E. Tosatti: Phys. Rev. B 73 (2006) 245116.

16) W. Kohn: Phys. Rev. 133 (1964) A171.

17) D. J. Scalapino, S. R. White, and S. Zhang: Phys. Rev. Lett. 68 (1992) 2830.

18) D. J. Scalapino, S. R. White, and S. Zhang: Phys. Rev. B 47 (1993) 7995.

19) B. Sriram Shastry and B. Sutherland: Phys. Rev. Lett. 65 (1990) 243.

20) B. Hetényi: J. Phys. Soc. Jpn. 81 (2012) 023701.

21) X. Zotos, H. Castella, and P. Prelovšek: in Correlated Fermions and Transport in Mesoscopic Systems, Proc. 31st Recontres de Moriond Conf. (Edition Frontieres, Paris, 1996).

22) S. Kirchner, H. G. Evertz, and W. Hanke: Phys. Rev. B 59 (1999) 1825 .

23) M. V. Berry: Proc. R. Soc. London, Ser. A 392 (1984) 45.

24) A. Shapere and F. Wilczek: Geometric Phases in Physics (World Scientific, Singapore, 1989).

25) J. Zak: Phys. Rev. Lett. 62 (1989) 2747.

26) R. Resta: Rev. Mod. Phys. 66 (1994) 899.

27) R. Resta: Phys. Rev. Lett. 80 (1998) 1800

28) R. Resta and S. Sorella: Phys. Rev. Lett. 82 (1999) 370.

29) R. Resta: Berry Phase in Electronic Wave Functions (Ecole Polytechnique Fédérale, Lausanne, 1996) Troisième Cycle Lecture Notes [http://ale2ts.ts.infn.it:6163/resta/publ/notes_trois.ps.gz].

30) F. H. L. Essler, H. Frahm, F. Göhmann, A. Klümper, and V. E. Korepin: The One-Dimensional Hubbard Model (Cambridge University Press, Cambridge, U.K., 2005).

31) B. Hetényi: J. Phys. A 42 (2009) 412003.

32) W. F. Brinkman and T. M. Rice: Phys. Rev. B 2 (1970) 4302.

33) P. Fazekas: Lecture Notes on Electron Correlation and Magnetism (World Scientific, Singapore, 1999). 